Supporting Information for

\title{
Record Low Thermal Conductivity of Polycrystalline Si Nanowire: Breaking the Casimir Limit by Severe Suppression of Propagons
}

\author{
Yanguang $\mathrm{Zhou}^{1}$ and Ming $\mathrm{Hu}^{1,2, *}$ \\ ${ }^{1}$ Aachen Institute for Advanced Study in Computational Engineering Science (AICES), RWTH \\ Aachen University, 52062 Aachen, Germany \\ ${ }^{2}$ Institute of Mineral Engineering, Division of Materials Science and Engineering, Faculty of \\ Georesources and Materials Engineering, RWTH Aachen University, 52064 Aachen,
} Germany

*Author to all whom correspondence should be addressed. E-Mail: hum@ghi.rwth-aachen.de (M.H.) 


\section{Construction of polycrystalline Si NWs}

The structure of polycrystalline $\mathrm{Si}$ NWs is generated by three-dimensional Voronoi tessellation method, which is quite successful way to study the mechanical, structure and thermal properties in material science ${ }^{1}$. For a set of seeds in a domain (the box of the NW in our case), the tessellation is defined by associating a set of random points (atoms with a random orientation in our simulations) in the box and is closer to a specific seed than any other seeds. For instance, to generate the polycrystalline Si NW in our paper, a set of seeds (the number of grains) is randomly selected in a simulation box (see Figure 1 in the paper) firstly. After that, the atomistic structure around each seed is generated following the diamond structure with random crystal orientation. Whether a generated atom around a certain seed belongs to this specific seed is determined by the fact that if the distance between this atom and this seed is larger than that between this atom and other seeds or not. Then, the polycrystalline Si NW can be built as shown in Figure 1c. One thing we have to mention is that some atoms in the interface region may be unreasonable. Therefore, atoms that are too close to each other at the grain boundaries are removed (the cutoff is $0.16 \mathrm{~nm}$ in our case since the nearest distance between atoms in diamond $\mathrm{Si}$ is $0.225 \mathrm{~nm}$, see the RDF figure below) before simulation. Then, the structure is relaxed at $300 \mathrm{~K}$ for $10 \mathrm{~ns}$ to reach a stable structure with zero pressure, using a NPT (constant particles, constant pressure and constant temperature) ensemble. After this periodic boundary relaxation, $1 \mathrm{~ns}$ running with free boundaries along the lateral direction is carried out to obtain the final stable and zero pressure polycrystalline Si NW. Figure S1 shows the temperature and total energy vs. time, from 
which we can find the system reaches equilibrium state after $8 \mathrm{~ns}$ relaxation run.
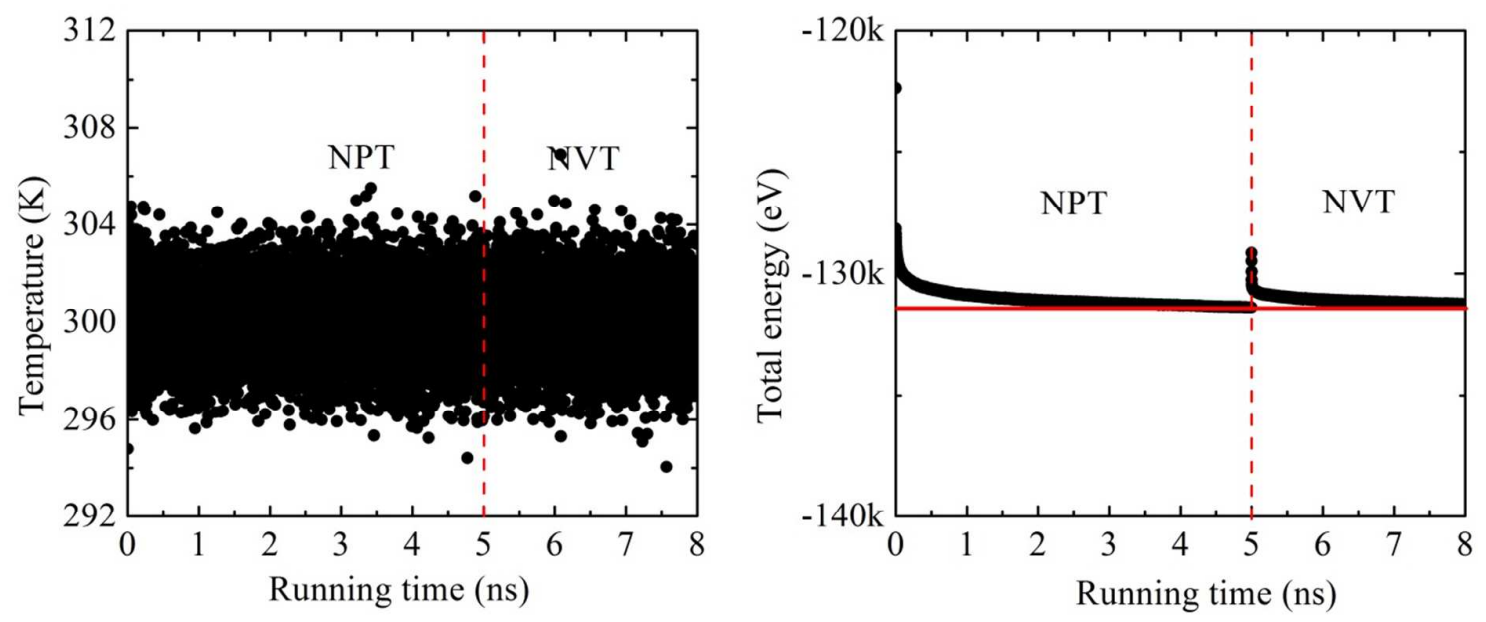

Figure S1. The temperature and energy evolution of the polycrystalline Si NW vs. time.

\section{Method to generate the amorphous Si NWs}

To obtain amorphous Si NW, we firstly build a periodic perfect Si structure with width of 10 unit cells and length of 300 unit cells. Then, the structure is melted at $5000 \mathrm{~K}$ for $2 \mathrm{~ns}$ using the NPT ensemble. The next procedure is to quench the structure from $5000 \mathrm{~K}$ to $300 \mathrm{~K}$ in a period of $8 \mathrm{~ns}$. Then, the structure is relaxed at $300 \mathrm{~K}$ for $500 \mathrm{ps}$ using the $N P T$ ensemble. Following that, the boundary condition is switched to free boundary, and the structure is then relaxed for about $2 \mathrm{~ns}$ at $300 \mathrm{~K}$. Figure S2 shows the temperature and total energy of the system vs. time. It is obviously found that the amorphous Si NW in our case is stable. Then, we fixed two atom layers at two ends along the longitudinal direction and generate the steady heat current flowing in our system as discussed in the main text. It has been proved that this 
method can generate a more or less realistic amorphous structure ${ }^{2}$.
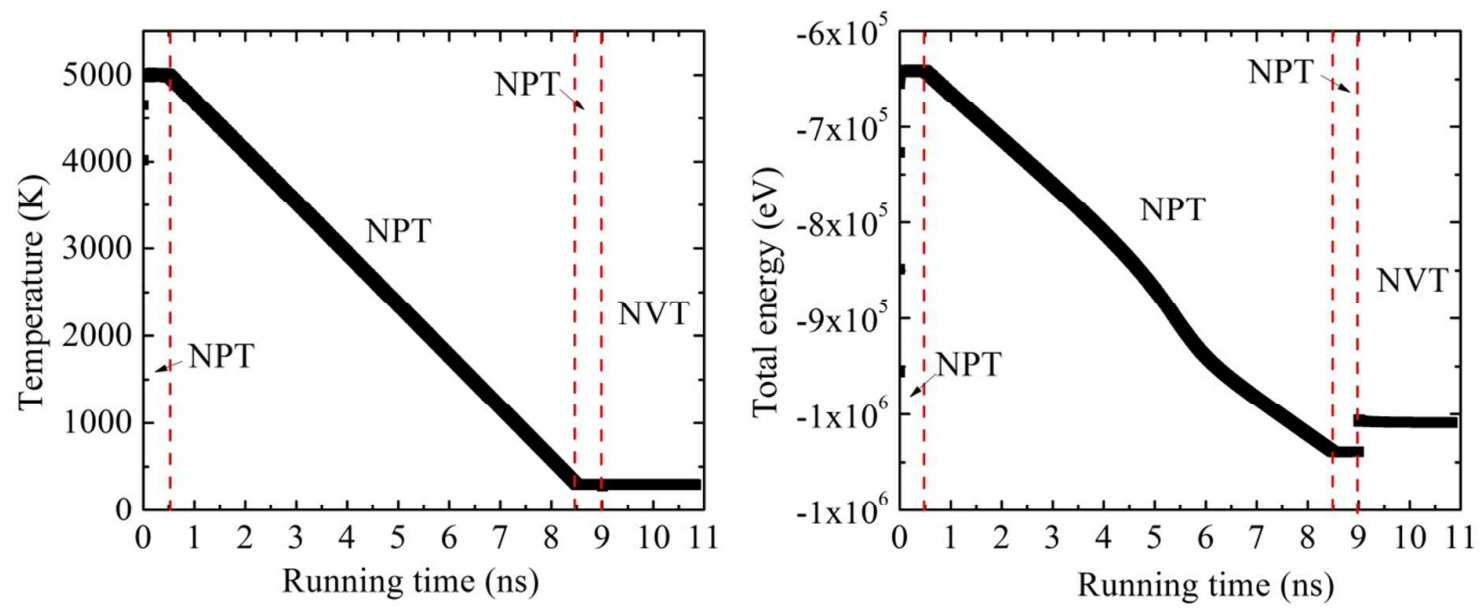

Figure S2. The temperature and energy evolution of the amorphous Si NW vs. time.

\section{Simulation details of GK-EMD}

In GK-EMD simulations, periodic boundary conditions are applied in all three directions. The system is relaxed in the NPT ensemble at $300 \mathrm{~K}$ for 500 ps to obtain the optimized structure, and then, the heat current in the $N V E$ ensemble (constant particles, constant volume and constant total energy) can be calculated using

$$
\mathbf{J}=\sum_{i}^{N} e_{i} \cdot \mathbf{v}_{i}+\frac{1}{2} \sum_{i, j ; i \neq j}^{N}\left(\mathbf{F}_{i j} \cdot \mathbf{v}_{i}\right) \cdot \mathbf{r}_{i j}+\sum_{i, j, k ; i \neq j \neq k}^{N}\left(\mathbf{F}_{i j k} \cdot \mathbf{v}_{i}\right) \cdot\left(\mathbf{r}_{i j}+\mathbf{r}_{i k}\right),
$$

where the $e_{i}$ is the energy of atoms, $\mathbf{v}_{i}$ is the velocity of atoms, $\mathbf{r}_{i j}$ is the distance between atom $i$ and $j, \mathbf{F}_{i j}$ and $\mathbf{F}_{i j k}$ are the two-body and three-body force, respectively. Then, the thermal conductivity can be derived using 


$$
k=\frac{1}{3 k_{B} T^{2} V} \int_{0}^{\infty}\langle\mathbf{J}(\tau) \cdot \mathbf{J}(0)\rangle d \tau
$$

in which, $k$ is the thermal conductivity, $k_{B}$ is the Boltzmann constant, $V$ is the volume of the system, $T$ is the system temperature and $\tau$ is the autocorrelation time. The angular bracket means the ensemble average. In Figure S3 we plot the thermal conductivity of bulk Si vs. autocorrelation time of 10 independent samples and the final thermal conductivity of bulk $\mathrm{Si}$ is calculated by averaging these ten cases. Our results show that the thermal conductivity of bulk $\mathrm{Si}$ is about $226.07 \pm 2.86 \mathrm{~W} / \mathrm{mK}$.

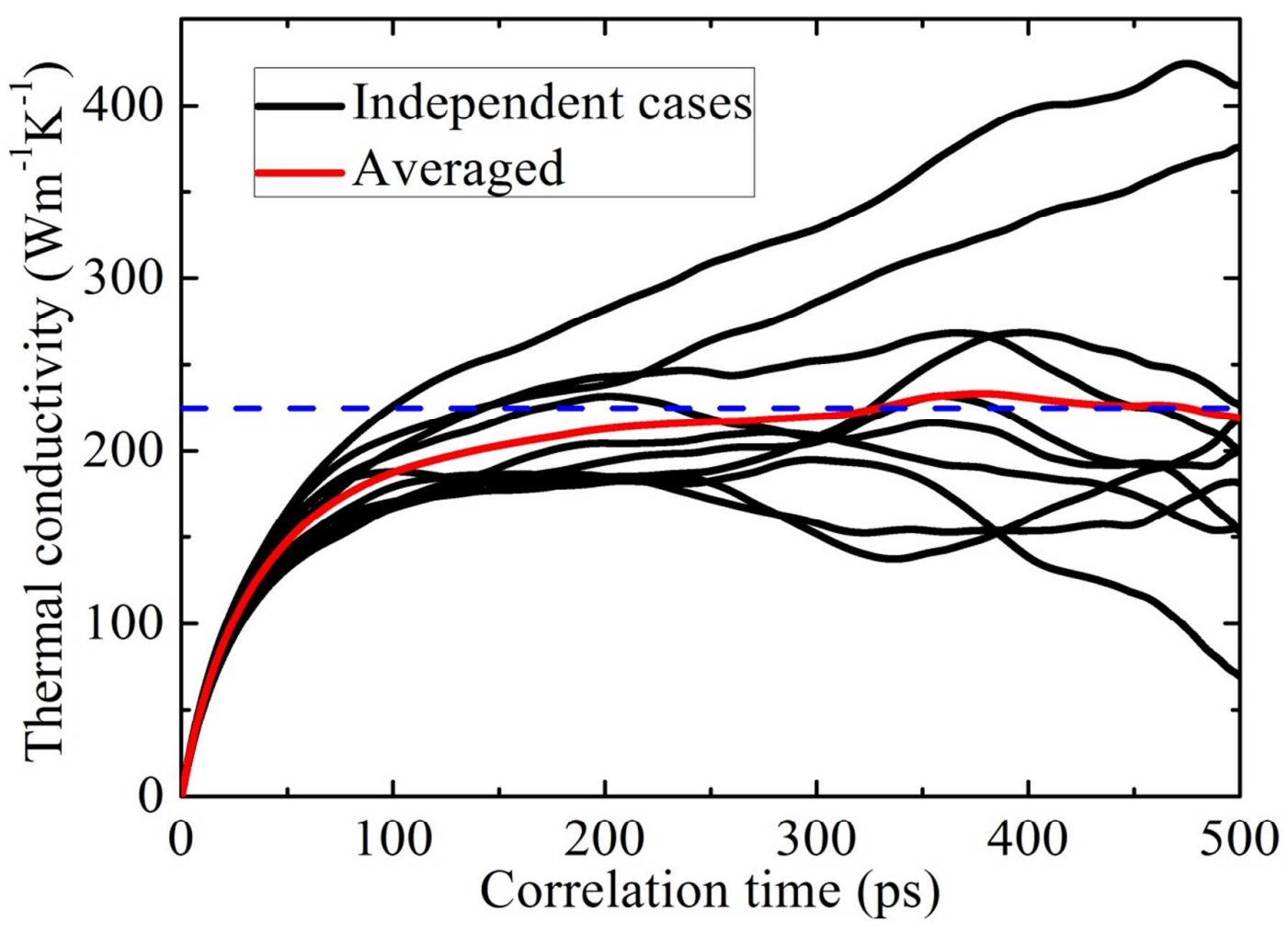

Figure S3. The thermal conductivity of bulk Si calculated by GK-EMD. 


\section{Thermal conductivity of crystalline Si NW calculated using full spectrum model}

Thermal conductivity of single crystalline Si NW with diameter of $4.35 \mathrm{~nm}$ (length $108 \mathrm{~nm}$ ) and (110) surface orientation calculated using full spectrum model ${ }^{3,4}$ with inputs from different empirical potentials and $a b$ initio calculation, is plotted in Figure S4. The root mean square of roughness is $0.15 \mathrm{~nm}$ in the full spectrum model, which is determined by various different orientation surface of Si NWs (Figure 5 in Ref. [3]). Holland model is used to calibrate our results with input from Ref. [5]. It is clearly seen that our MD results using the Tersoff potential agree well with our full spectrum $a b$ initio predictions.

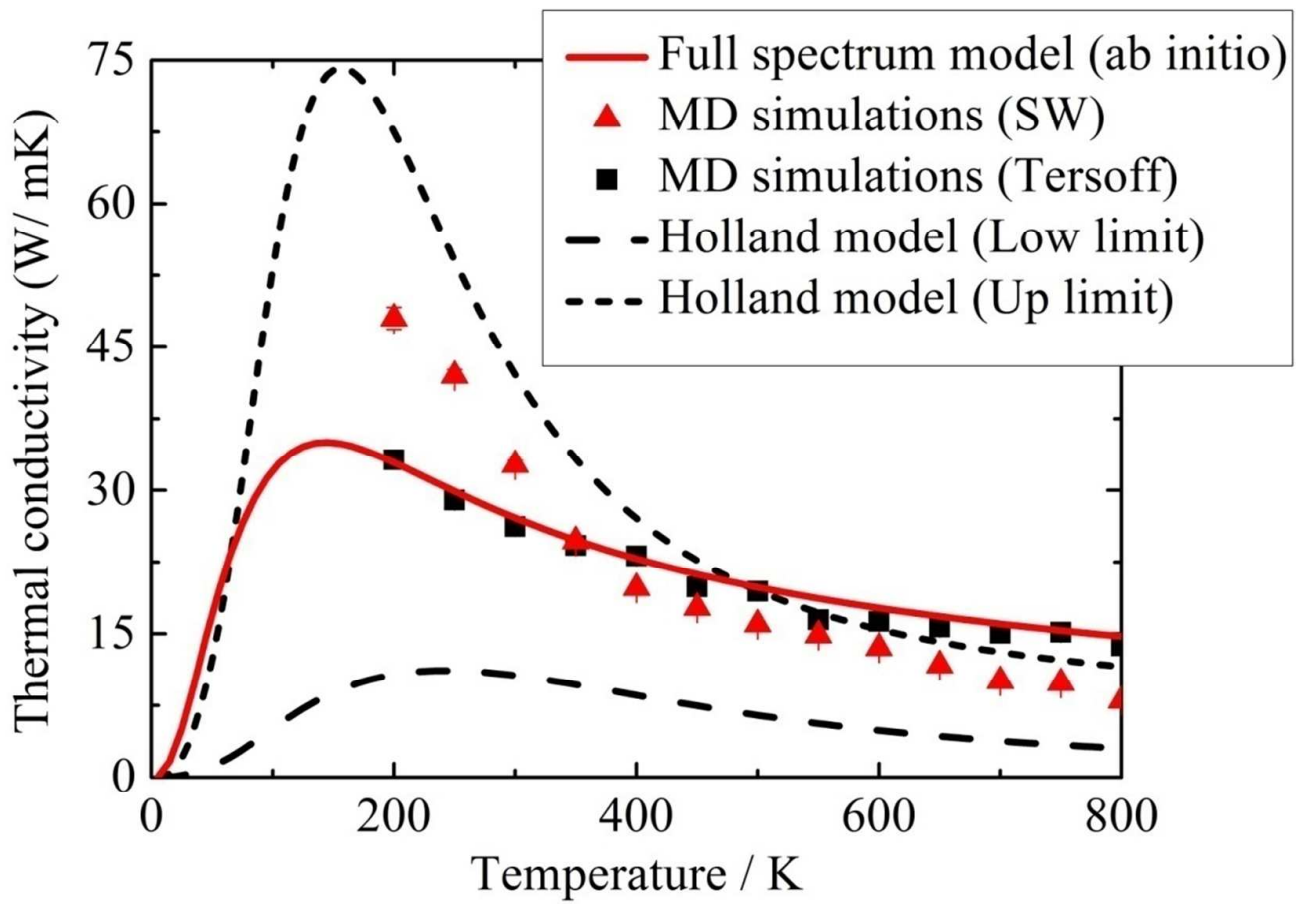

Figure S4. Thermal conductivity of single crystalline Si NW with diameter of $4.35 \mathrm{~nm}$ (length $108 \mathrm{~nm}$ ) and (110) surface orientation calculated using full spectrum model (solid line) with inputs from $a b$ initio calculation. 


\section{Method to obtain the effective group velocity of vibrational modes for disordered systems}

In the disorder systems such as amorphous $\mathrm{Si}$ and polycrystalline $\mathrm{Si}$ NWs in our paper, all vibrational modes will be located at the $\Gamma$ point, and then, the group velocity $\mathbf{v}_{g}(\mathbf{q}=\mathbf{0})=d \omega / d \mathbf{q}$ of vibrational modes will be zero (except the three acoustic mode corresponding to $\omega=0$ ), if we take the whole system as the unit cell as Lakin and McGaughey did in Ref. [6]. However, as discussed by He et al. ${ }^{7}$, we know that the low frequency modes keep the propagating character and $\omega(\mathbf{q}=\mathbf{0})$ flattens out only for $\mathbf{q} \approx \mathbf{0}$. Therefore, following He et al., we define the effective group velocity $\mathbf{v}_{g}(\mathbf{q}=\mathbf{0})=\Delta \omega / \Delta \mathbf{q}$ without taking the limit for $\mathbf{q} \rightarrow \mathbf{0}$. 50 replication of the unit cell for the amorphous Si NW and 30 replication of the unit cell for polycrystalline Si NW, which corresponds to 50 and 30 wave vectors, respectively, are used to generate the effective group velocity by the PHNONPY software ${ }^{8}$.

\section{Relaxation time and effective group velocity of vibrational modes for the four types of structures}

The effective group velocity calculated using the method mentioned above and the relaxation time computed by Eq. (2) is plotted in Figure S5 and Figure S6, respectively. For perfect Si NW, the effective group velocity and the relaxation time are found to decrease globally with respect to bulk $\mathrm{Si}$ due to the phonon-boundary scattering. For amorphous $\mathrm{Si}$ NW and polycrystalline $\mathrm{Si} \mathrm{NW}$, the effective group velocity of these vibrations in the low frequency 
(smaller than $2 \mathrm{THz}$ ) are found to be in the same amplitude of bulk Si and perfect Si NW, while that for the vibrations in the middle and high frequency region (above $2 \mathrm{THz}$ ) decreases largely. Meanwhile, the relaxation time of the vibrations for amorphous $\mathrm{Si}$ NW and polycrystalline Si NW in the frequency region larger than $1.5 \mathrm{THz}$ decreases dramatically with respect to the bulk $\mathrm{Si}$ and perfect $\mathrm{Si}$ NWs. Specifically, the relaxation time of polycrystalline Si NW is even smaller than that of amorphous Si NW, which means a quite strong scattering in polycrystalline Si NW.
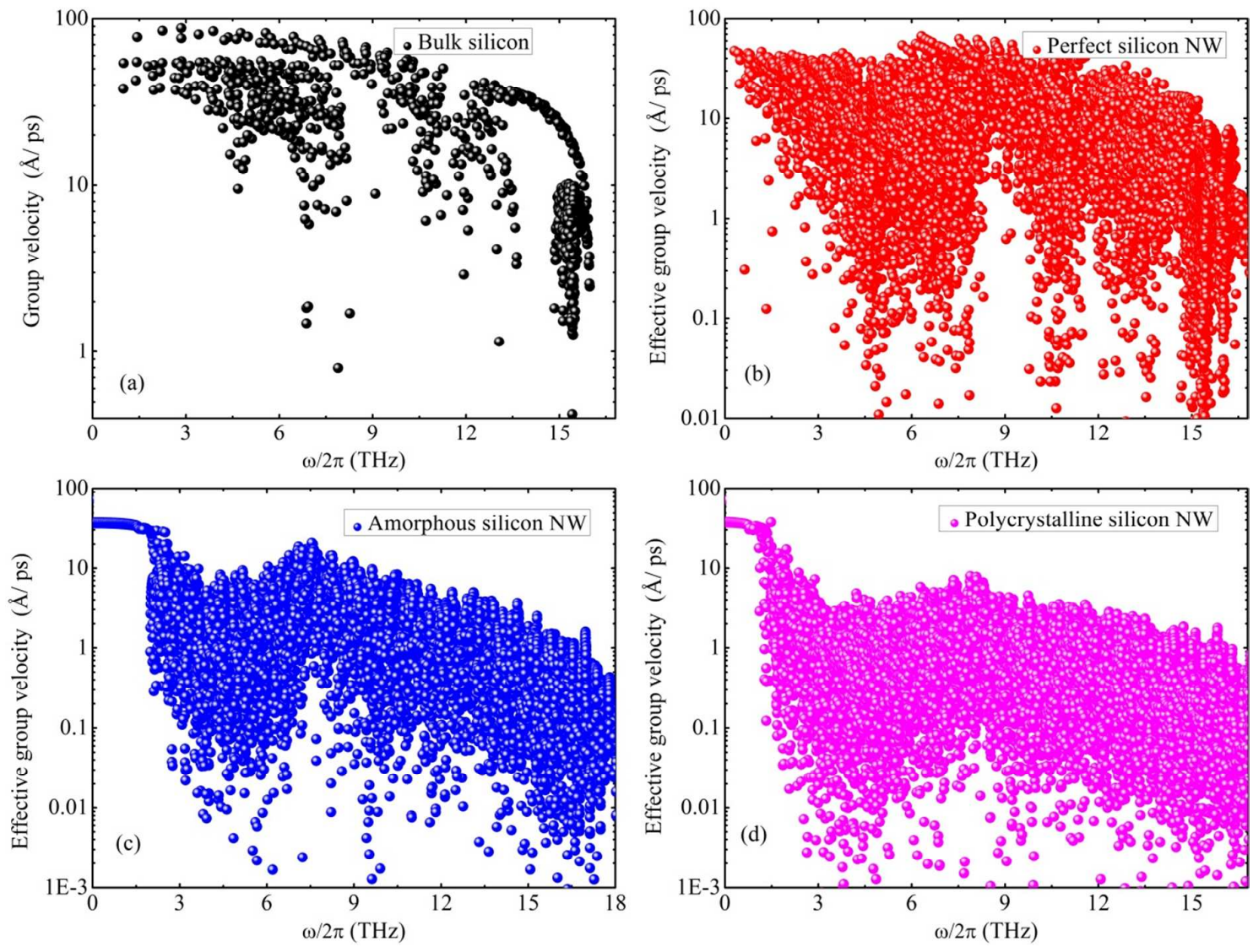

Figure S5. The group velocity of (a) bulk Si, (b) perfect Si NW, (c) amorphous Si NW and (d) polycrystalline Si NW. 

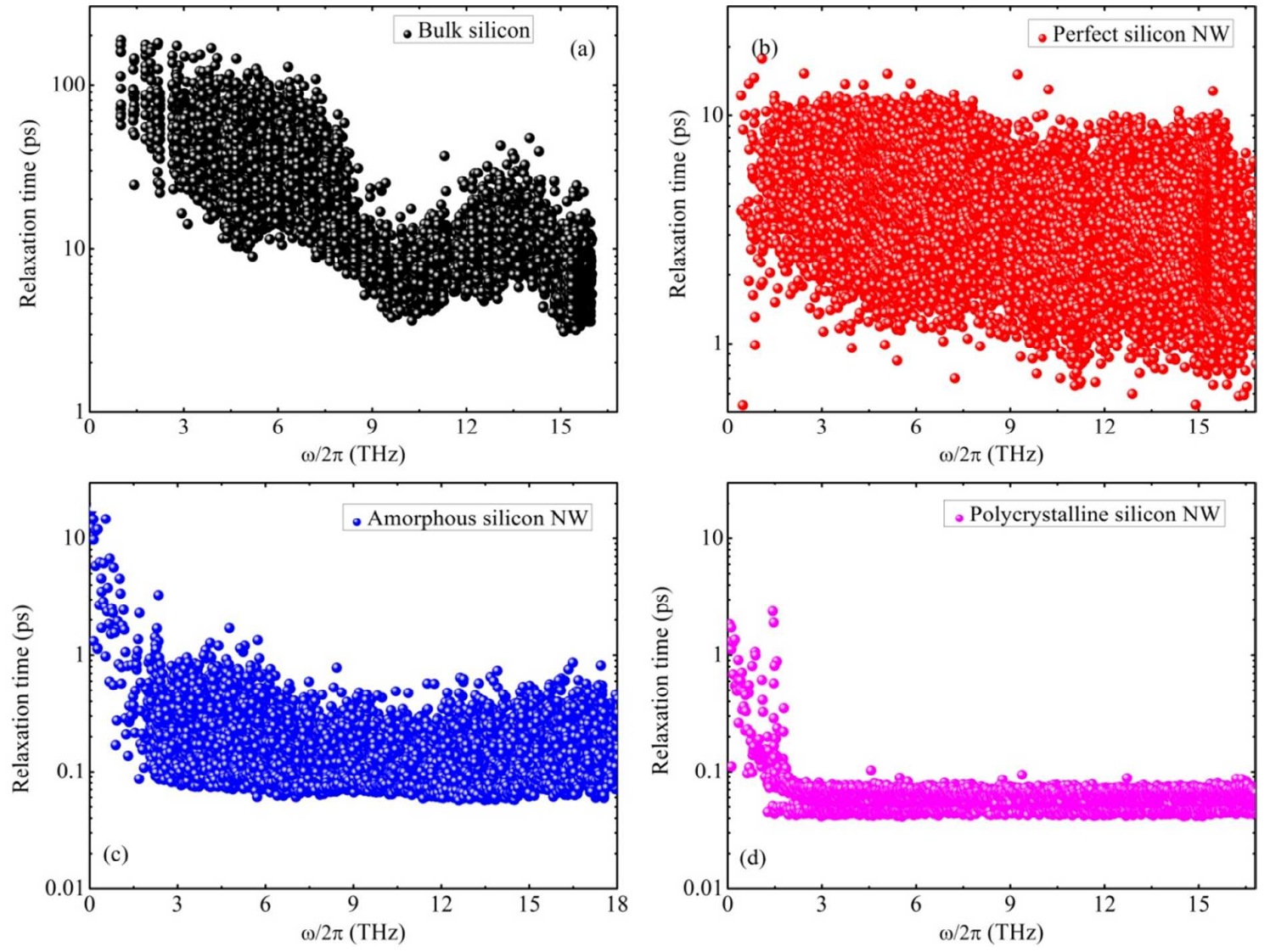

Figure S6. The relaxation of (a) bulk Si, (b) perfect Si NW, (c) amorphous Si NW and (d)

polycrystalline Si NW.

\section{RDF of four model structures}

In order to determine the AVD as defined in our paper, we calculate the RDF of these four kinds of model structures (Figure S7) with a cut-off parameter of $0.3 \mathrm{~nm}$. From the results, we can easily find the nearest distance between atoms, or equivalently, the AVD, should be the value around $0.22 \mathrm{~nm}$. 


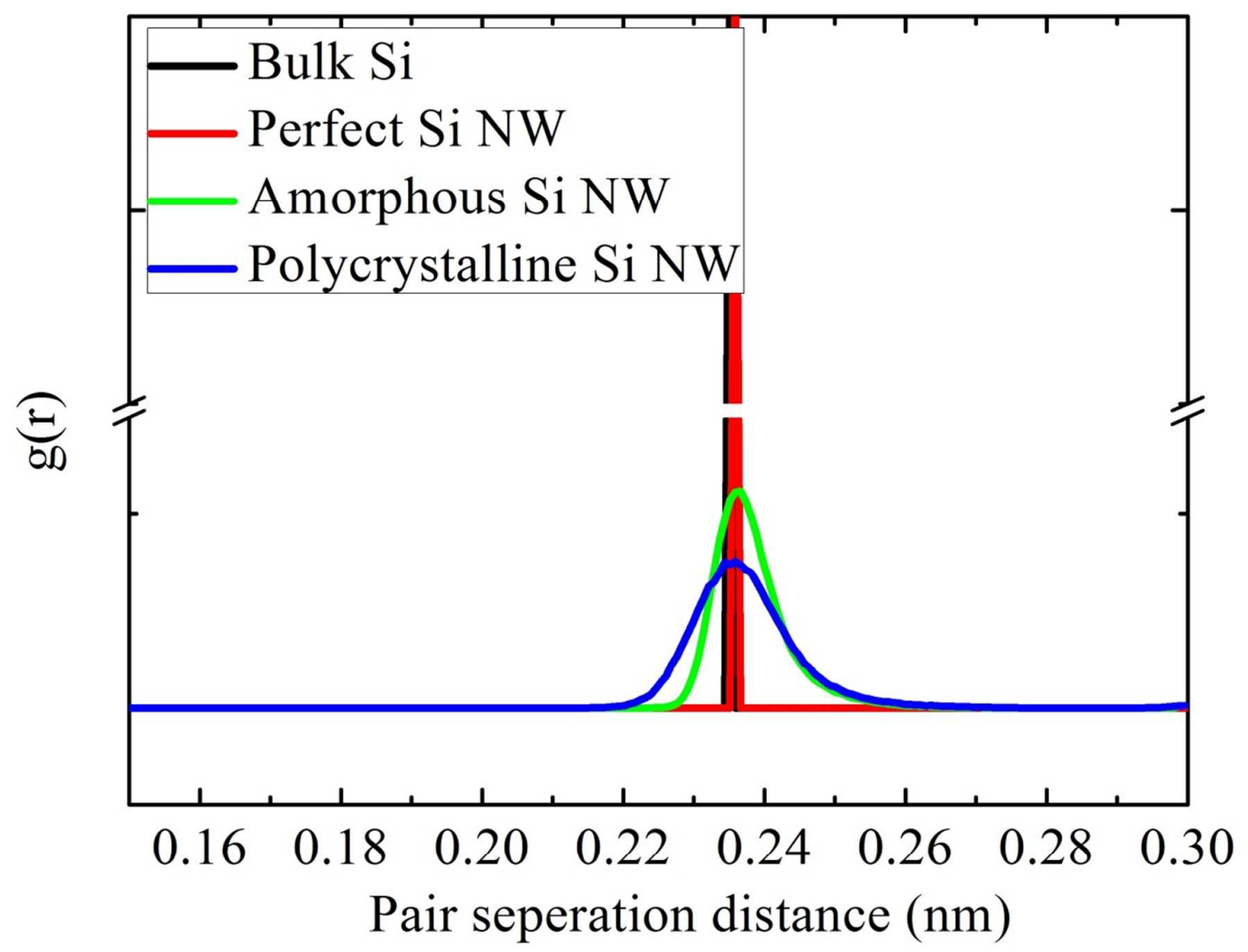

Figure S7. The radial distribution function of four types of Si structures. 


\section{References}

1. Yamakov, V.; Wolf, D.; Phillpot, S. R.; Mukherjee, A. K.; Gleiter, H. Nature mater. 2003, 3, 43-47.

2. France-Lanord, A.; Blandre, E.; Albaret, T.; Merabia, S.; Lacroix, D.; Termentzidis, K. J. Phys.2014, 26, 055011.

3. Zhou, Y.; Chen, Y.; Hu, M. Sci. Rep. 2016, 6, 24903.

4. Aksamija, Z.; Knezevic, I. Phys. Rev. B 2010, 82, 045319.

5. Holland, M. G. Phys. Rev. 1963, 132, 2461.

6. Larkin, J. M.; McGaughey, A. J. Phys. Rev. B 2014, 89, 144303.

7. He, Y.; Donadio, D.; Galli, G. Appl. Phys. Lett. 2011, 98, 144101.

8. Togo, A.; Tanaka, I. Scr. Mater. 2015, 108, 1-5. 